Original Research Paper

\title{
High-Protein Solid-State Symbiotic Fermentation of Sauce Residue for Probiotic Feed
}

\author{
${ }^{1,3+}$ Xinhe Zhao, ${ }^{2+}$ Zhuangzhuang He, ${ }^{1}$ Wenwei Lu, ${ }^{3}$ Yubin Zhao, ${ }^{1}$ Yuanda Song and ${ }^{1 *}$ Xiaojie Ren \\ ${ }^{1}$ Colin Ratledge Center for Microbial Lipids, School of Agriculture Engineering and Food Science, \\ Shandong University of Technology, Zibo, Shandong, China \\ ${ }^{2}$ School of Agriculture Engineering and Food Science, Shandong University of Technology, Zibo, Shandong, China \\ ${ }^{3}$ Luzhou Bio-Chem Technology Limited, Linyi, Shandong, China
}

\author{
Article history \\ Received: 22-07-2020 \\ Revised: 08-10-2020 \\ Accepted: 09-10-2020 \\ Corresponding Author: \\ Xiaojie Ren \\ Colin Ratledge Center for \\ Microbial Lipids, School of \\ Agriculture Engineering and \\ Food Science, Shandong \\ University of Technology, \\ Zibo, Shandong, China \\ Email: renxiaojie2020@163.com \\ ${ }^{+}$Contributed equally to this \\ work and should be considered \\ co-first authors
}

\begin{abstract}
The abuse of antibiotics in animal feed has caused a series of problems in livestock breeding, meat products and the natural environment. In this study, sauce residue was used as the main raw material that includes $25.98 \%$ of dry-based crude protein, $3.91 \%$ of $\mathrm{NaCl}, 16.28 \%$ of crude fat, $11.06 \%$ of the ash and the multi-strain solid-state symbiotic fermentation technology was used to increase the amount of probiotics and the crude protein content of feed. Meanwhile, the addition of functional probiotics improved the feed palatability, while reducing the use of antibiotics further improved the cycle utilization of soy sauce residue. This study systematically optimized the symbiotic conditions of two probiotics, Lactobacillus bulgaricus and Saccharomyces cerevisiae. This study defined viable cell count and crude protein content as the core targets for analysis and evaluation. Firstly, the optimal carbon source concentration, the temperature and the inoculation process of symbiotic fermentation were determined by single factors; then the symbiotic fermentation conditions (inoculation volume, material-liquid ratio, fermentation time) were optimized by orthogonal test. The results showed that $40 \mathrm{~g} / \mathrm{L}$ glucose/lactose $(50 / 50 \%)$ was the best carbon source for sauce residue fermentation. The optimum fermentation conditions were a ratio of material to water of 1:0.8 and an inoculation volume of $15 \%(\mathrm{v} / \mathrm{w})$ and a fermentation duration of $48 \mathrm{~h}$. Meanwhile, the study found that multistep temperature control could effectively improve the corresponding targets. The inoculation with Saccharomyces cerevisiae first and then with Lactobacillus bulgaricus after $24 \mathrm{~h}$, boosted the probiotic quantity and protein content. In optimized conditions, probiotic viable count reached $7.2 \times 10^{8} \mathrm{CFU} \cdot \mathrm{mL}^{-1}$ and the crude protein content increased from $25.98 \%$ (dry content) to $38.86 \%$, which is $49.6 \%$ increase compared with the raw material. This research could provide a reference for the development of high-protein probiotic feed using sauce residue.
\end{abstract}

Keywords: Sauce Residue, Probiotic Feed, Symbiosis Fermentation, Saccharomyces cerevisiae, Lactobacillus bulgaricus

\section{Introduction}

In recent years, the abuse of antibiotics in animal feed has severely affected animals, meat products and the environment (Kumar et al., 2005). The long-term feeding of antibiotics to livestock and poultry has caused severe damage to the micro-ecological environment of their digestive tracts (Gu and Peng, 2015). Human health is further affected by human consumption of these livestock and poultry products (Barton, 2000). Therefore, developing probiotic feeds to replace antibiotics could regulate the health of the animals by boosting their immunity. This is significant for the development of quality livestock and poultry meat products.

Sauce residue (soy sauce residue) is a by-product of soy sauce brewing (Chen et al., 2017). The global annual output of soy sauce residue is more than 100 million tons per year and this causes increasingly serious 
environmental pollution problems (Gong et al., 2018). Some manufacturers dispose it by landfill, which results in soil salinization and serious soil pollution (Duan et al., 2019). Sauce residue is rich in nutrients such as protein, fat, etc., which can breed microorganisms (Chen et al., 2017). A high crude fiber content and some antinutritional factors of sauce residue make it difficult to be absorbed by the digestive tracts of animals. So, it is difficult to use original sauce residue directly as feed (Asada et al., 2010; Murasawa et al., 2013). With the higher requirement of environmental protection, the reuse of sauce residue is urgently needed.

Orthogonal experimental design is a design method to study multi-factors and multi-levels. According to the orthogonality, some representative points are selected from the comprehensive test. It can judge the significant degree of the influence of factors on the test index. Meanwhile, it can find out the optimal level of test factors and the optimal combination within the test range and estimate the size of the test error. But it cannot examine the interaction between factors when the purpose of the experiment is mainly to find a good horizontal combination. Therefore, in this study, orthogonal experiments were selected to find the optimal symbiotic fermentation conditions with fewer experiments and to find out the significance of symbiotic fermentation conditions on the viable count and crude protein content.

Microbial fermented feed, especially probiotic fermented feed, can produce a large number of metabolites that are beneficial to animals, decomposing the antinutritional factors and greatly improving the nutritional value of the feed (Wang et al., 2019). The ability of probiotics to adjust the micro-ecological balance of animal intestines could promote the growth of animals, enhance their immunity and thus reduce the need for antibiotics (Zhao and Dai, 2020). This study used sauce residue as raw materials to establish two probiotic symbiotic fermentation modes of Lactobacillus bulgaricus and Saccharomyces cerevisiae. The fermentation parameters were optimized based on the symbiotic fermentation technology. This study aimed to increase the quality of fermented probiotic feed and thus provide theoretical reference and data support for the development of high-protein probiotic feed from sauce residue.

\section{Materials and Methods}

\section{Materials}

Saccharomyces cerevisiae and Lactobacillus bulgaricus were preserved at the Comprehensive Laboratory of Food Science and Engineering, Shandong University of Technology, Zibo, China. They are stored in a slant preservation in a refrigerator at $4{ }^{\circ} \mathrm{C}$. The raw material used for the experiment was fresh sauce residue provided by Boshan Zhengtang Brewing Factory in Zibo City,
China. The water content of the sauce residue was $66.7 \%$, the $\mathrm{pH}$ value was 6.35 , the dry-based crude protein content was $25.98 \%$ (and the wet-based content was $5.97 \%$ ), the $\mathrm{NaCl}$ content was $3.91 \%$, the crude fat content was $16.28 \%$ and the ash content was $11.06 \%$. DeManRogosa-Sharpe (MRS) medium and Yeast Extract Peptone Dextrose (YPD) medium were used for the seed liquid culture of Lactobacillus bulgaricus and Saccharomyces cerevisiae respectively. Bengal red medium was used to determine the number of viable cell count.

\section{Methods}

\section{Selection and Addition of Carbon Source}

The carbon source was selected from glucose, maltose, lactose, sucrose and glucose/lactose (50/50\%). Twenty (20) $\mathrm{g} / \mathrm{L}$ of the carbon source and $500 \mathrm{~g} / \mathrm{L}$ of sauce residue were added in each culture and sterilized at $121^{\circ} \mathrm{C}$ for 20 min. Saccharomyces cerevisiae and Lactobacillus bulgaricus were inoculated at 1:1 ratio with inoculation volume of $10 \%(\mathrm{v} / \mathrm{w})$ and fermented at $32^{\circ} \mathrm{C}$ (Yan and $\mathrm{He}$, 2012; Zhai et al., 2018). After 48 h, the effective viable count and crude protein content were measured.

Then the optimal concentration of the carbon source was determined by using $10,20,30,40$ and $50 \mathrm{~g} / \mathrm{L}$ respectively for further fermentation.

\section{Research based on Temperature Regulation}

Single temperature control: Fermentation was carried out for $48 \mathrm{~h}$ at $28,30,32,34$ and $36^{\circ} \mathrm{C}$ respectively, after which the fermentation products were sampled and analyzed.

Two-step temperature rise control: The temperature was controlled at $30^{\circ} \mathrm{C}$ for the first $24 \mathrm{~h}$ of the fermentation process and at $34^{\circ} \mathrm{C}$ for the next $24 \mathrm{~h}$, after which the fermentation products were sampled and analyzed.

Multi-step temperature rise control: The temperature was controlled at $30^{\circ} \mathrm{C}$ for the first $12 \mathrm{~h}(0-12 \mathrm{~h})$ of the fermentation process, at $32^{\circ} \mathrm{C}$ for the next $12 \mathrm{~h}(12-24$ h), at $34^{\circ} \mathrm{C}$ for the third duration of $12 \mathrm{~h}(24-36 \mathrm{~h})$ and at $36^{\circ} \mathrm{C}$ for the final $12 \mathrm{~h}(36-48 \mathrm{~h})$, after which the fermentation products were sampled and analyzed.

\section{Research based on the Inoculation Process}

Simultaneous inoculation: Saccharomyces cerevisiae and Lactobacillus bulgaricus were inoculated at 1:1 ratio at the same time; the fermentation conditions were the same as for the 'multi-step temperature rise control' and the fermentation products were sampled and analyzed after $48 \mathrm{~h}$.

Lactobacillus bulgaricus was inoculated first, which was followed after $24 \mathrm{~h}$ of culturing by the inoculation of Saccharomyces cerevisiae; the fermentation conditions were the same as for the 'multi-step temperature rise control' and the fermentation products were sampled and analyzed after a total inoculation time of $48 \mathrm{~h}$. 
Table 1: Factors and levels of orthogonal test

\begin{tabular}{llll}
\hline Levels & Material-liquid ratio & Inoculation volume $(\%)$ & Fermentation time $(\mathrm{h})$ \\
\hline 1 & $1: 0.8$ & 10 & 36 \\
2 & $1: 1$ & 15 & 48 \\
3 & $1: 1.2$ & 20 & 60 \\
\hline
\end{tabular}

Saccharomyces cerevisiae was first inoculated, which was followed after $24 \mathrm{~h}$ of culturing by the inoculation of Lactobacillus bulgaricus. The fermentation conditions were the same as for the 'multi-step temperature rise control' and the fermentation products were sampled and analyzed after a total inoculation time of $48 \mathrm{~h}$.

\section{Optimization of the Fermentation Conditions}

Using the effective viable count, crude protein and the $\mathrm{pH}$ value of the fermentation product as the detection index, the experiments were carried out at various values of the solid-liquid ratios (1:0.6, 1:0.8, 1:1, 1:1.2, 1:1.4), inoculation amounts $(5,10,15$ and $20 \%)$, fermentation times $(24$ h, 36 h, 48 h, 60 h) were carried out. Other culture conditions were adopted such as the multi-step temperature, $40 \mathrm{~g} / \mathrm{L}$ glucose/lactose $(50 / 50 \%)$ as the carbon source and the inoculation by Saccharomyces cerevisiae first. Based on the above single factor test results, the $\mathrm{L}^{9}\left(3^{4}\right)$ orthogonal test was carried out. The orthogonal test factors and levels are shown in Table 1. From the orthogonal test results, double index analysis was performed to optimize the fermentation conditions.

\section{Measurement Methods of Related Indicators}

The drying differential weight method was used to measure the moisture content, the Soxhlet extraction method was used to determine the crude fat content, the burning weight method was used to determine the ash content and the Kjeldahl determination was used for crude protein measurement (Zhang et al., 2017). The $\mathrm{NaCl}$ content of sauce residue was determined by silver nitrate titration. The number of viable cells was determined by the dilution plate counting method (Hou et al., 2018). All data were analyzed by one-way Analysis of Variance (ANAVA), compared by Duncan multiple comparisons at $\mathrm{P}<0.05$ using SPSS and all the experiments were carried out in parallel and expressed as the means \pm Standard Deviation (SD). This part has been revised in the manuscript.

\section{Results and Analysis}

\section{Carbon Source Significantly Affected Symbiotic Fermentation}

Sauce residue contains lots of crude fiber but lack small molecules of sugar which Lactobacillus bulgaricus and Saccharomyces cerevisiae can utilize directly (Zhang et al., 2017). So, an external carbon source is needed to improve cell growth. Several commonly used fermentable sugars were added into the medium, including glucose, maltose, lactose, sucrose and glucose/lactose $(50 / 50 \%)$. The effective viable count of probiotics was determined after culturing for $48 \mathrm{~h}$. The results are shown in Figure 1. At $8.32 \times 10^{7} \mathrm{CFU} \cdot \mathrm{mL}^{-1}$, the effective viable count of probiotics in the glucose/lactose $(50 / 50 \%)$ culture group was highest. In the control group (no external carbon source was added), the number of viable probiotics was only $1.7 \times 10^{7}$ $\mathrm{CFU} \cdot \mathrm{mL}^{-1}$. Therefore, with carbon source added, the best expression of the viable probiotics obtained was about 5-fold that of the control group, showing that the combination of lactose and glucose could significantly improve the growth of Lactobacillus bulgaricus and Saccharomyces cerevisiae. However, when utilizing glucose and lactose as single carbon sources, the number of viable cells obtained were $3.30 \times 10^{7}$ and $7.00 \times 10^{7}$ $\mathrm{CFU} \cdot \mathrm{mL}^{-1}$ respectively. Both of these single carbon sources expressed lower viable cells than the combination of lactose and glucose culture. Meanwhile, when maltose and sucrose were separately added, the number of effective viable cells in each case was lower than that when any of glucose and lactose was used as the carbon source. In this study, the cells showed preference for lactose. In fact, after one molecule of lactose was used by the microorganisms, one molecule of glucose and one molecule of galactose were obtained. While Lactobacillus bulgaricus prefers to make use of galactose, Saccharomyces cerevisiae prefers to use glucose directly. Therefore, the addition of glucose/lactose $(50 / 50 \%)$ as the optimal carbon source was beneficial to the growth of both Saccharomyces cerevisiae and Lactobacillus bulgaricus.

The best carbon source of glucose/lactose $(50 \% / 50 \%)$ was further optimized by varying the concentrations and the results were shown in Fig. 2. When the concentration of glucose/lactose $(50 / 50 \%)$ was $40 \mathrm{~g} / \mathrm{L}$, the effective viable count of probiotics was $3.47 \times 10^{7} \mathrm{CFU} \cdot \mathrm{mL}^{-1}$, which was the highest obtained for any concentration. The number of viable probiotics in Fig. 2 shows a tendency to rise first as the concentration of carbon source rises, until it reaches a maximum value and then slowly begins to decline, indicating that when the concentration of the carbon source was too low, the requirements for probiotics growth were not met, especially as Saccharomyces cerevisiae required a higher carbon source. When the concentration of the carbon source was $50 \mathrm{~g} / \mathrm{L}$, the effective viable count of 
probiotics decreased, indicating that this mass concentration was too high and inhibited the growth and reproduction of the probiotics. When the mass concentration of glucose + lactose $(1: 1)$ was $40 \mathrm{~g} / \mathrm{L}$, the crude protein content of the supernatant of the fermentation broth was $3.94 \%$. At the same concentration, the viable count of Saccharomyces cerevisiae was also the highest, reaching $2.01 \times 10^{7}$ $\mathrm{CFU} \cdot \mathrm{mL}^{-1}$. The growth trend of the viable count of Saccharomyces cerevisiae was basically consistent with the growth trend of the crude protein content, which showed that yeast as the main source of fermented cell protein could increase the crude protein content of fermented feed. When the mass concentration was 30 $\mathrm{g} / \mathrm{L}$, the viable count of Lactobacillus bulgaricus was $1.73 \times 10^{7} \mathrm{CFU} \cdot \mathrm{mL}^{-1}$, its highest level, indicating that this concentration was the most suitable for the growth and reproduction of Lactobacillus bulgaricus. However, the number of Saccharomyces cerevisiae was only $0.88 \times 10^{7}$ $\mathrm{CFU} \cdot \mathrm{mL}^{-1}$ at this concentration. The main reason was that the excessively high lactic acid produced by the action of Lactobacillus bulgaricus on lactose drastically reduced the $\mathrm{pH}$ of the symbiotic environment, which inhibited the growth of yeast and ultimately led to low total probiotics. Therefore, the final experimental results showed that the optimal mass concentration of glucose + lactose $(1: 1)$ was $40 \mathrm{~g} / \mathrm{L}$.

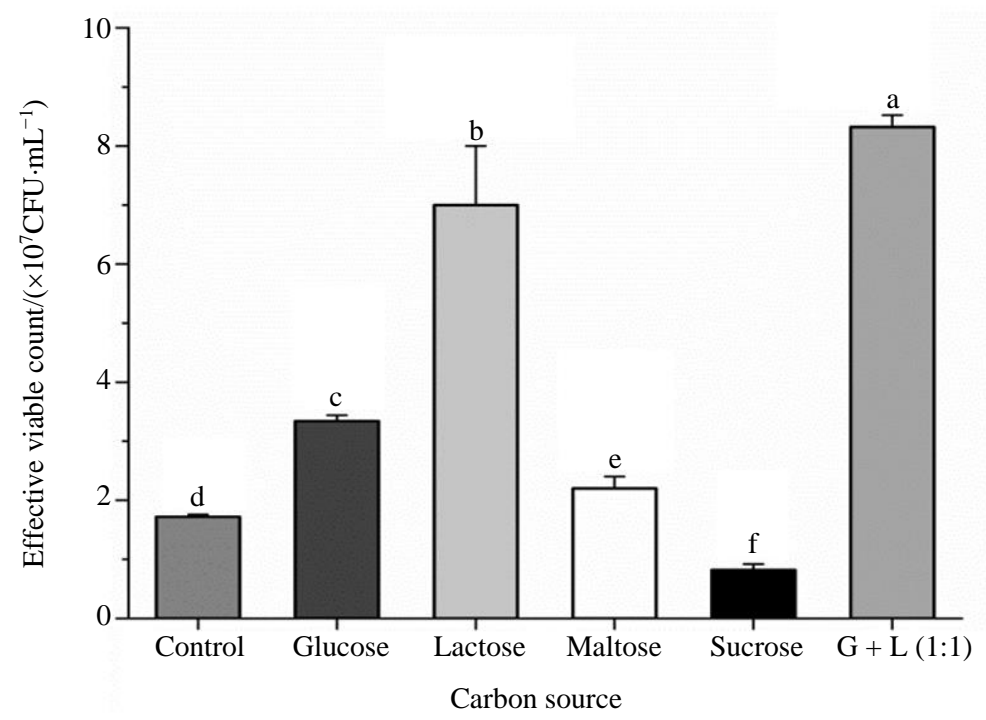

Fig. 1: Effects of different carbon sources on the growth of probiotics in fermented sauce. Control means no carbon source was added; $G+L(1: 1)$ represents carbon source with a glucose to lactose ratio of $1: 1$. The different letters indicate that the comparison of each peak value with the data of its neighboring detection points yielded a significant difference $(\mathrm{P}<0.05)$
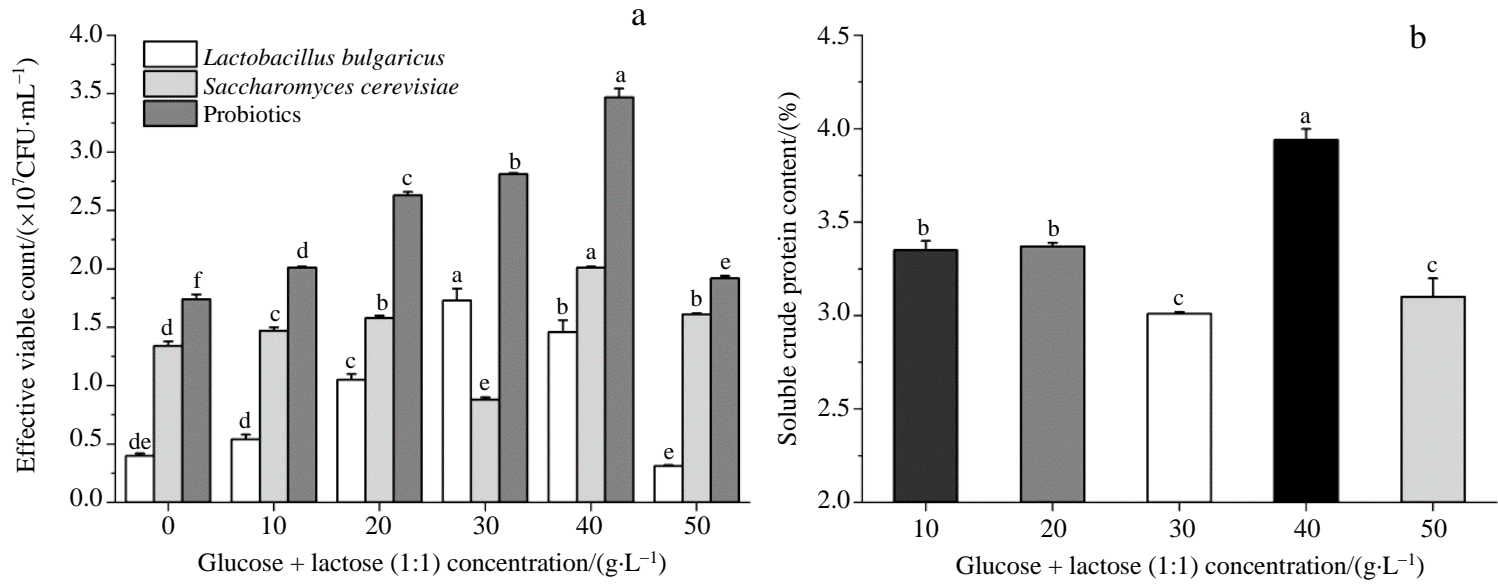

Fig. 2: Effects of different concentrations of glucose + lactose (1: 1) on fermented sauce 


\section{Multi-Step Temperature-Controlled Improved Symbiotic Culture}

Generally, the suitable growth temperature of Saccharomyces is $28 \sim 30^{\circ} \mathrm{C}$, while that of the Lactobacillus is $36 \sim 40^{\circ} \mathrm{C}$ (Han et al., 2014). Since the optimal growth temperatures of Lactobacillus bulgaricus and Saccharomyces cerevisiae differ significantly, the multi-step temperature method was used to control the fermentation process, mainly to avoid the rapid propagation of the Lactobacillus, which would have led to its dominance and the production of a large amount of lactic acid, causing the $\mathrm{pH}$ to drop and inhibiting the growth of yeast (Han et al., 2014). In fact, various temperature control methods were tried and the results are shown in Fig. 3. When the temperature was kept constant at $32^{\circ} \mathrm{C}$, the number of effective viable count of probiotics was $1.93 \times 10^{7}$ $\mathrm{CFU} \cdot \mathrm{mL}^{-1}$. In the multi-step temperature-controlled culture, the effective viable count, at $1.88 \times 10^{7} \mathrm{CFU} \cdot \mathrm{mL}^{-1}$, was similar to that of the $32^{\circ} \mathrm{C}$ constant-temperature culture, but the crude protein content $(7.88 \%)$ was significantly higher than those of the other groups. The crude protein content of the $32^{\circ} \mathrm{C}$ constant-temperature culture, at just $6.81 \%$, was of course lower than that of the multi-step temperature culture group. In fact, over low temperature or over high temperature both inhibited cells growth. For example, in the culture of 28 and $36^{\circ} \mathrm{C}$, the effective viable count and crude protein content were both lower than those of the $32^{\circ} \mathrm{C}$ culture and the multi-step temperature controlled fermentation. The rate of cell growth and the crude protein content of the 30 and $34^{\circ} \mathrm{C}$ constanttemperature cultures fell between the corresponding values for the $2836^{\circ} \mathrm{C}$ culture and the multi-step temperaturecontrolled group. It seems that single temperature control was not suitable for the growth of symbiotic strains in the cultures. In the multi-step temperature-controlled group, the number of effective viable count of Lactobacillus bulgaricus was highest $\left(1.51 \times 10^{7} \mathrm{CFU} \cdot \mathrm{mL}^{-1}\right)$, which indicated that this method was beneficial to the growth of Lactobacillus bulgaricus. Therefore, the optimized culture plan was to select a suitable temperature for Saccharomyces cerevisiae growth for a period of time and then raise the temperature to allow the bacteria to multiply in a large amount and to reduce the impact of lactic acid production by Lactobacillus bulgaricus. The experimental results showed that temperature-controlled fermentation can be used as the key parameter in the optimization of symbiotic fermentation technology and the multi-step temperature control was the best choice for the symbiotic fermentation of Saccharomyces cerevisiae and Lactobacillus bulgaricus.

\section{Staggered Inoculation Process Effectively Promoted Cell Growth}

In symbiotic fermentation, the characteristics of different strains determine if the symbiosis status of the strains are different when utilizing different inoculation sequences. Figure 4 showed the effective live count and the crude protein content when the sauce residue was inoculated first with Lactobacillus bulgaricus and then inoculated with Saccharomyces cerevisiae after $24 \mathrm{~h}$ of fermentation (group A); when it was inoculated first with Saccharomyces cerevisiae and then inoculated with Lactobacillus bulgaricus after $24 \mathrm{~h}$ (group B); and when simultaneously inoculated (group C) by the two bacteria. The total number of viable probiotic in group B was significantly higher than in the other groups $\left(7.8 \times 10^{7}\right.$ $\mathrm{CFU} \cdot \mathrm{mL}^{-1}$ ) and the number of Lactobacillus bulgaricus was also highest $\left(6.6 \times 10^{7} \mathrm{CFU} \cdot \mathrm{mL}^{-1}\right)$. The effective viable count in group A was only $3.8 \times 10^{7} \mathrm{CFU} \cdot \mathrm{mL}^{-1}$. Interestingly, the $\mathrm{pH}$ of the above two groups did not show much difference after $48 \mathrm{~h}$. Therefore, except that the lactic acid produced by Lactobacillus bulgaricus could affect the initial growth of the Saccharomyces cerevisiae, a symbiotic enzymatic system plays a key role in such fermentation. For the simultaneously inoculated process (group C), the number of viable probiotic fell between the values for the two staggered inoculation methods. However, the crude protein content for the simultaneous inoculation method, at $26.81 \%$, was higher than for the two staggered inoculation. But it is the method of inoculation first with Saccharomyces cerevisiae and then with Lactobacillus bulgaricus after $24 \mathrm{~h}$ (group B), that can be considered the best method, because it expressed a high value for both the effective cell count and crude protein content.

\section{Optimization of the Symbiotic Fermentation Conditions}

\section{Material-Liquid Ratio Experiments}

The material-liquid ratio is one of the key factors that affect solid-state fermentation. Using the effective viable count, $\mathrm{pH}$ and crude protein as indicators, different material-liquid ratios were optimized. As shown in Fig. 5, when the material-liquid ratio in the sauce residue medium was 1:0.8, the total effective viable count reached a maximum of $3.77 \times 10^{8} \mathrm{CFU} \cdot \mathrm{mL}^{-1}$ and both the viable counts of Saccharomyces cerevisiae and Lactobacillus bulgaricus were significantly higher than the corresponding values for the other ratios. When the material-liquid ratio was $1: 1.4$, the total effective viable count was as low as $0.45 \times 10^{8} \mathrm{CFU} \cdot \mathrm{mL}^{-1}$, just $12 \%$ of the viable count for the ratio 1:0.8. In a high material-liquid ratio medium, the nutrients are diluted and the porosity of the matrix is reduced, which in turn reduces the gas concentration of the medium and then inhibits the growth of microorganisms (Zhan et al., 2015). However, if the material-liquid ratio is too low, the swelling degree of the matrix is reduced, which decreases the concentration of the nutrients and the fluidity of the bacteria, thereby also inhibiting the growth of microorganisms (Zhan et al., 
2015). For the material-liquid ratio of 1:0.8, the crude protein content of the dry-base of the fermented feed reached a maximum of $36.89 \%$, which is about $57 \%$ higher than the crude protein content for the ratio 1:1.4 $(23.52 \%)$. Therefore, the material-liquid ratio of 1:0.8 was defined as the optimal ratio.
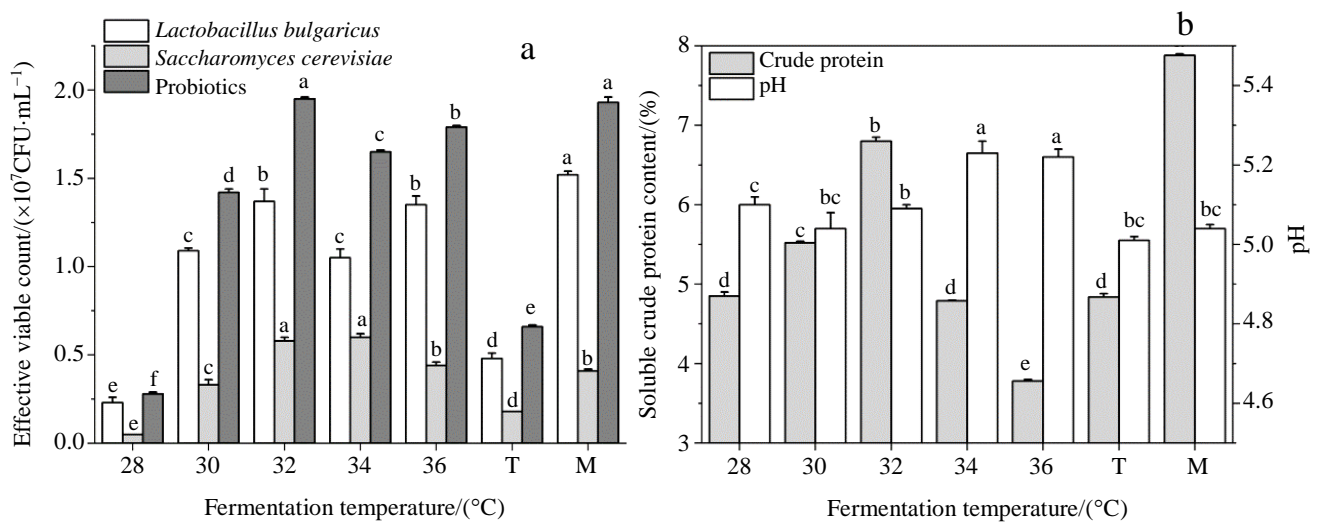

Fig. 3: Effects of temperature control on the fermentation of sauce residue. T represents two-step temperature rise control; M represents multi-step temperature rise control
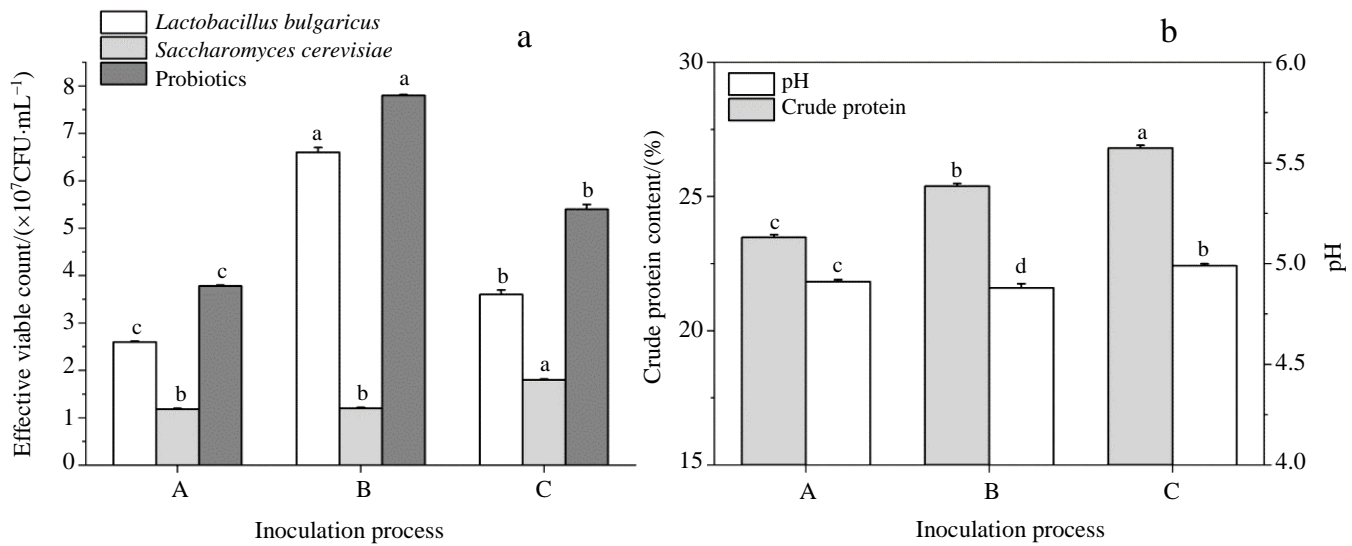

Fig. 4: Effect of probiotic inoculation process on fermented sauce residue. Group A represents sauce residue inoculation first with Lactobacillus bulgaricus first and then with Saccharomyces cerevisiae after culturing for $24 \mathrm{~h}$; Group B represents inoculation with Saccharomyces cerevisiae first and then with Lactobacillus bulgaricus after culturing for 24 h; Group C represents simultaneous inoculation
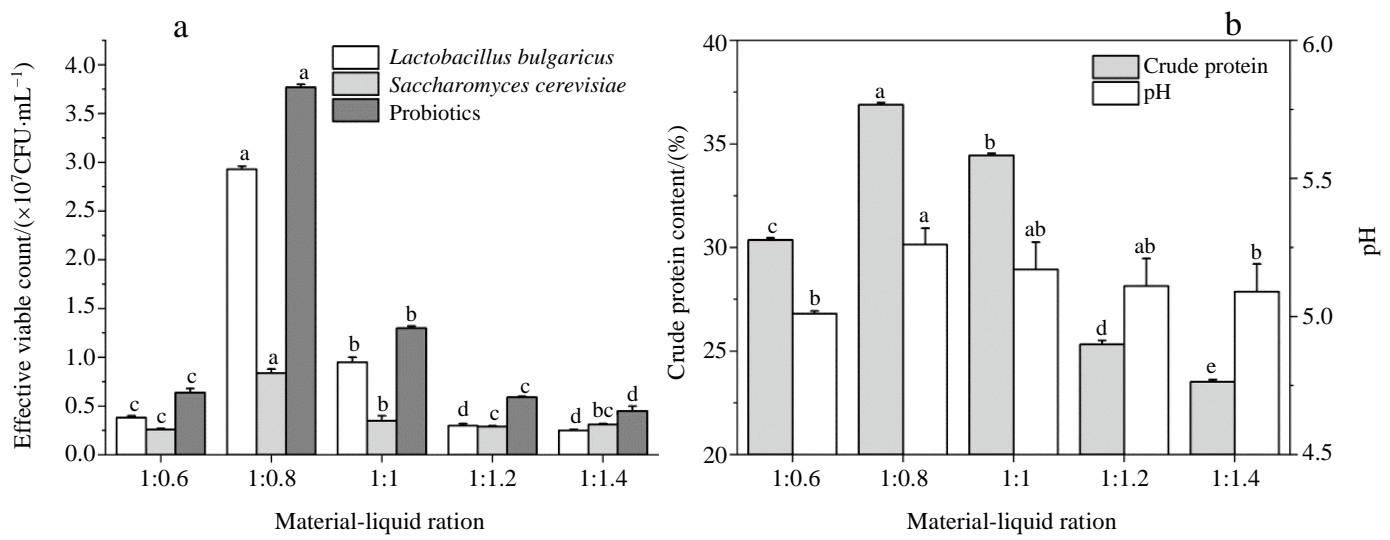

Fig. 5: Effect of material-liquid ratio on fermented sauce residue 


\section{The Inoculation Volume Affected Fermentation Conversion Rate and the Effective Viable Count of Probiotics}

The inoculation amount can directly affect the fermentation cycle and conversion rate. The viable count, $\mathrm{pH}$ value and the crude protein content of the drybase were tested for the optimization of the inoculation amount; the results are shown in Fig. 6. When the inoculation amount was $15 \%$, the total effective viable count of the ferment $\left(1.93 \times 10^{8} \quad \mathrm{CFU} \cdot \mathrm{mL}^{-1}\right)$ was significantly higher than for the other inoculation doses and the number of live Lactobacillus bulgaricus was also highest $\left(1.53 \times 10^{8} \mathrm{CFU} \cdot \mathrm{mL}^{-1}\right)$. At the same inoculation amount was $15 \%$, the dry-base crude protein content of the fermented feed reached the highest value of $40.42 \%$. But when the inoculation amount was $20 \%$, the effective viable count of the probiotics in the fermented feed started to decrease. This is because when the inoculation amount was too high, the nutrients in the medium were quickly consumed, which inhibited the growth of microorganisms. In addition, the inoculation amount was positively related to the $\mathrm{pH}$ value of the fermentation broth. This may be because the inoculum amount was too large, which led to insufficient oxygen and nutrients and to premature decay of probiotics, resulting in $\mathrm{pH}$ increase. Meanwhile, Saccharomyces cerevisiae produced ethanol under anaerobic condition, which inhibited the growth of Lactobacillus bulgaricus and the production of lactic acid. In addition, the excessive inoculation of Saccharomyces cerevisiae also affected the fermentation of Lactobacillus bulgaricus. Therefore, based on the factors mentioned above, $15 \%$ was selected as the optimal inoculation volume.

\section{Effect of Fermentation Time}

With increase in symbiotic fermentation time, the total number of viable probiotics, the number of viable Lactobacillus bulgaricus and the dry-base crude protein content all showed increasing trends first and then decreasing trends (Fig.7). After $48 \mathrm{~h}$, the effective viable count of the probiotics in the fermented feed had reached its highest at $1.2 \times 10^{8} \mathrm{CFU} \cdot \mathrm{mL}^{-1}$ and the viable count of Lactobacillus bulgaricus was $9.5 \times 10^{7}$ $\mathrm{CFU} \cdot \mathrm{mL}^{-1}$. After the same time, the dry-based crude protein content was $34.45 \%$. Then, as the fermentation time increased, the number of viable probiotics began to decrease. This may be because as the fermentation time got too long, the nutrients reduced and the bacteria began to decay, leading to a rise in $\mathrm{pH}$ and the decrease in the number of viable probiotics. However, the number of viable yeast and $\mathrm{pH}$ value showed continuously increasing trends. The growth cycle of yeast was longer than that of Lactobacillus bulgaricus. The $\mathrm{pH}$ increased after autolysis and decay of the lactobacillus in the late stage, making it suitable for the growth of yeast. Due to the lack of nutrients in the late stage, yeast decomposed proteins as carbon and nitrogen sources for its growth, resulting in the decrease in the crude protein content in the late stage. Therefore, $48 \mathrm{~h}$ was the most suitable cultivation time for the symbiotic fermentation (Fig.7).

\section{Orthogonal Test}

According to the single-factor experiment on the fermentation conditions (material-water ratio, inoculation amount, fermentation time), the optimal factor level was selected for the $\mathrm{L}^{9}\left(3^{4}\right)$ orthogonal experiment and the range analysis of two indices was carried out for the orthogonal test results (Table 2). Comparison of range sizes in Table 2 shows that the factors that affected the total effective viable count were inoculation amount (B) $>$ material-liquid ratio (A) > fermentation time $(\mathrm{C})>$ error (D), in that order; however, the order of the factors that affected the crude protein content was materialliquid ratio $(\mathrm{A})>$ fermentation time $(\mathrm{C})>$ inoculation amount (B) > error (D).
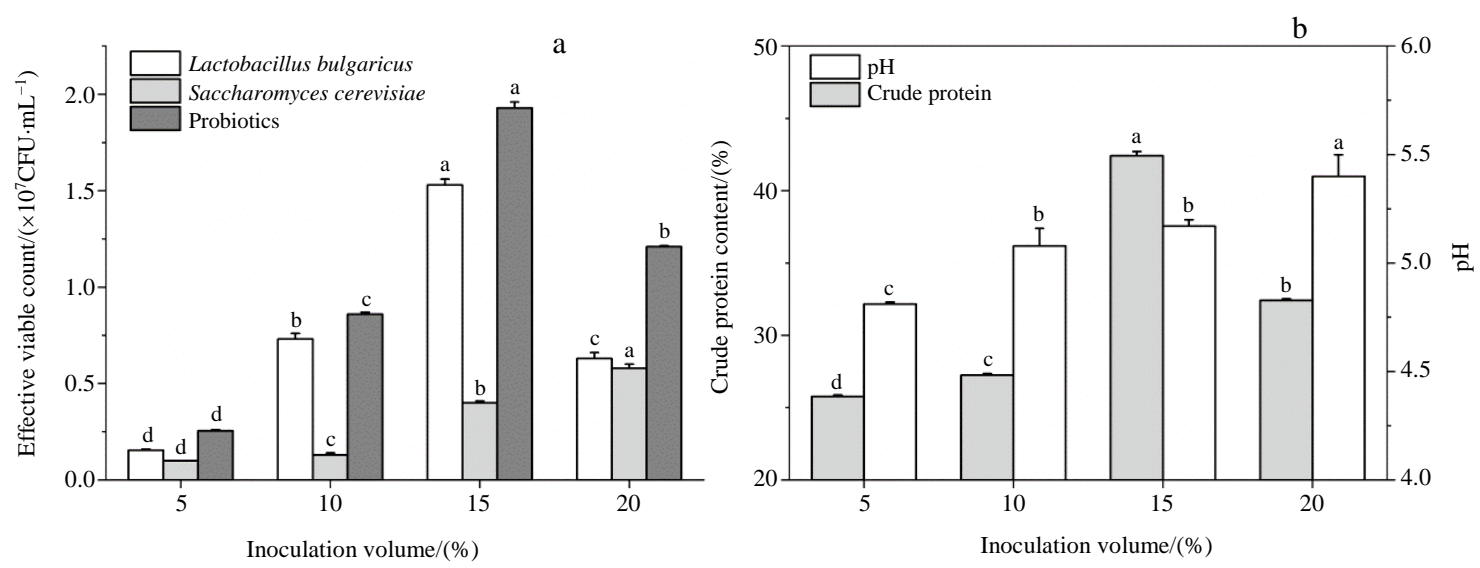

Fig. 6: Effect of probiotic inoculation on fermented sauce residue 

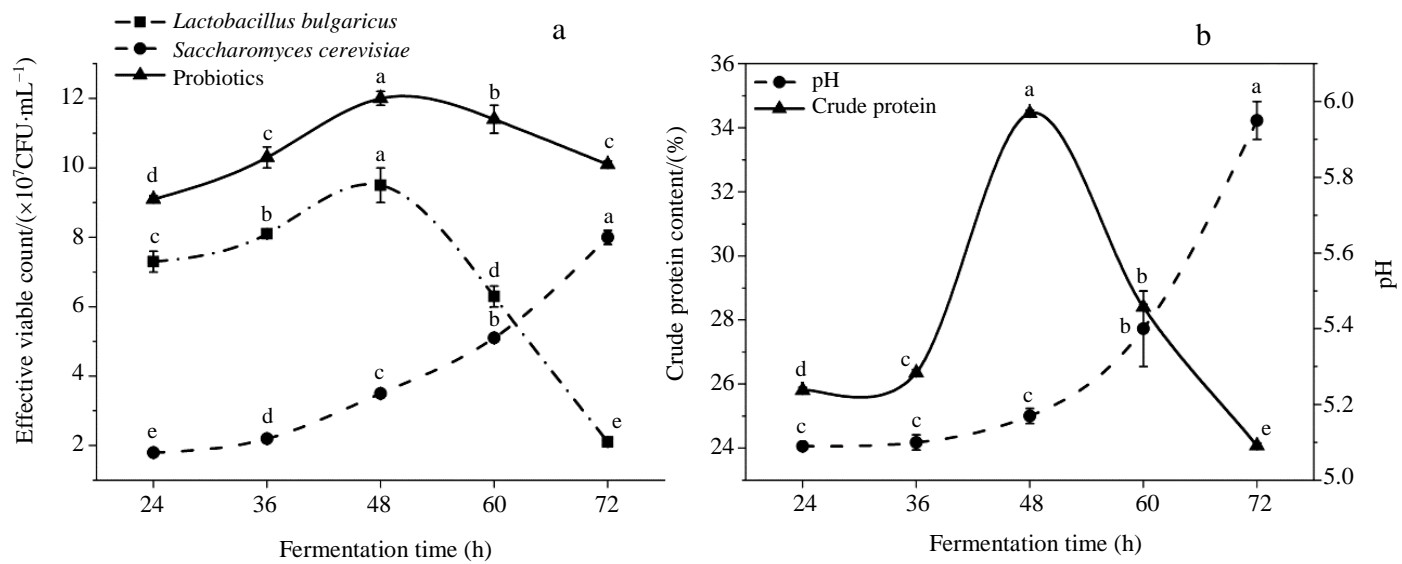

Fig. 7: Effect of fermentation time on fermented feed from sauce residue

Table 2: Results of the orthogonal test

\begin{tabular}{lcccccc}
\hline Test group & $\begin{array}{l}\text { Material-liquid } \\
\text { ratio A }\end{array}$ & $\begin{array}{l}\text { Inoculation } \\
\text { volume B }\end{array}$ & $\begin{array}{l}\text { Fermentation } \\
\text { time C }\end{array}$ & $\begin{array}{l}\text { Empty } \\
\text { column D }\end{array}$ & $\begin{array}{l}\text { Effective viable } \\
\text { count/ }\left(10^{8} \mathrm{CFU}_{\mathrm{mL}} \mathrm{mL}^{-1}\right)\end{array}$ & $\begin{array}{l}\text { Crude } \\
\text { protein } / \%\end{array}$ \\
\hline 1 & 1.00 & 1.00 & 1.00 & 1.00 & 4.20 & 30.72 \\
2 & 1.00 & 2.00 & 2.00 & 2.00 & 7.20 & 38.86 \\
3 & 1.00 & 3.00 & 3.00 & 3.00 & 3.80 & 29.29 \\
4 & 2.00 & 1.00 & 2.00 & 3.00 & 3.00 & 27.38 \\
5 & 2.00 & 2.00 & 3.00 & 1.00 & 5.30 & 20.93 \\
6 & 2.00 & 3.00 & 1.00 & 2.00 & 3.20 & 36.67 \\
7 & 3.00 & 1.00 & 3.00 & 2.00 & 4.10 & 3.11 \\
8 & 3.00 & 2.00 & 1.00 & 3.00 & 4.20 & 30.68 \\
9 & 3.00 & 3.00 & 2.00 & 1.00 & 5.00 & \\
$\mathrm{k}_{1}$ & 5.07 & 3.77 & 3.87 & 4.83 & & \\
$\mathrm{k}_{2}$ & 3.83 & 5.57 & 5.07 & 4.83 & & \\
$\mathrm{k}_{3}$ & 4.43 & 4.00 & 4.40 & 3.67 & & \\
$\mathrm{R}$ & 1.23 & 1.80 & 1.20 & 1.17 & & \\
$\mathrm{k}_{1}$ & 32.96 & 31.59 & 26.55 & 29.44 & & \\
$\mathrm{k}_{2}$ & 25.04 & 31.30 & 32.31 & 32.12 & & \\
$\mathrm{k}_{3}$ & 31.82 & 26.93 & 30.96 & 28.26 & & \\
$\mathrm{R}$ & 7.91 & 4.66 & 5.76 & 3.86 & & \\
\hline
\end{tabular}

This shows that the inoculation amount and the materialliquid ratio had the greatest impact on the effective viable count and crude protein content, respectively. The optimal level was determined according to the value of the single factor $\mathrm{K} 1$ and the optimal combination of fermentation conditions was finally determined as A1B2C2: That is the material-liquid ratio was 1:0.8, The inoculation amount was $15 \%$, the fermentation time was $48 \mathrm{~h}$, the effective viable number of probiotics could reach $7.20 \times 10^{8}$ $\mathrm{CFU} \cdot \mathrm{mL}^{-1}$ and the dry-based crude protein content could reach $38.86 \%$. After the verification test, the effective viable count was $6.90 \times 10^{8} \mathrm{CFU} \cdot \mathrm{mL}^{-1}$ and the dry-based crude protein content was $36.54 \%(\mathrm{P}<0.05)$.

\section{Discussion}

As the main by-product of soy sauce production, about 5.5 million tons of sauce residue was produced per year in China (2014) (Shu et al., 2015). The sauce residue is rich in protein (25-30\%), crude fiber (13$25 \%)$, crude fat $(7-10 \%)$, minerals $(8-16 \%)$ and other nutrients (Gong et al., 2018; Shu et al., 2015; Zhang et al., 2017). Therefore, it is extremely easy to rot and stink and its use is restricted (He et al., 2014). Many studies were devoted to the secondary extraction of obtain fat, crude protein, dietary fiber, soy isoflavones and other nutrients from sauce residue (Chen et al., 2015; 2014; He et al., 2014; Shu et al., 2015), but the extraction cost was relatively high and the extraction process could affect product quality and cause secondary pollution. There were also studies on the simple development of sauce residue into feed or fertilizer (Cui et al., 2014; Jing et al., 2016), but the feed has poor palatability and can easily cause salt poisoning etc. (Gong et al., 2018). In recent years, research on micro-ecological feed has developed rapidly. The fermentation of feed materials in the presence of probiotics not only improves the nutritional level and degrades the anti-nutritional factors 
of the feed, but it also improves the intestinal flora of the animal and produces a variety of probiotic metabolites, which boosts animal immunity (Zhao and Dai, 2020). The raw material for brewing soy sauce contains more protein, which is suitable for the growth of microbial flora. In addition, the sauce residue is rich in isoflavones, which improve immunity, improve lactation, promote growth and improve the quality of livestock and poultry products (Sun et al., 2016). Therefore, the fermentation of sauce residues with various probiotics not only utilizes the nutrients of the sauce residues, but also makes full use of the fermented materials to improve feed palatability. The use of fermented feed could ultimately reduce the need for antibiotics in animal feed and improve the health of animal intestines. Zhang et al. (2017) used Rhodospirillum rubrum to ferment soy sauce residue into microbial feed, which increased the nutritional content of the soy sauce residue and reduced the amount of toxic substances. Peng et al. (2013) used Aspergillus niger to degrade hemicellulose in sauce residue and the degradation rate reached $84.28 \%$ in 30 days.

In this study, sauce residue was used as raw materials for symbiotic fermentation and after the fermentation, the dry-based crude protein content of the biological feed was potentially $38.86 \%$, which is a $49.6 \%$ increase compared with the raw material. Relevant research also showed that microbial fermentation could improve the nutrient level of sauce residue. Bian et al. (2012) inoculated Geotrichum candidum, Candida tropicalis, Aspergillus niger (1:1:2) in sauce residue, which increased the crude protein content after fermentation by 23.09\%. However, most studies on fermented feed from sauce residue were concerned only with increasing the crude protein content, while neglecting the problem of the bad odor and poor palatability of sauce residue feed. Fermentation of sauce residue in the presence of probiotics not only increases the protein content and the number of live probiotics, but also produces more organic acids such as lactic acid, which gives the sauce residue a unique flavor and significantly boosts its attraction for animals. The metabolites and liquid produced by Lactobacillus bulgaricus have a strong bacteriostatic effect on pathogenic microorganisms. Lactobacillus bulgaricus and its metabolites contain more Superoxide Dismutase (SOD), which can regulate the immune function of animals (Yu et al., 2018; Zhao and Dai, 2020). Therefore, feed fermented in the presence of Lactobacillus bulgaricus not only has high nutritional value and utilization rate, but also is extremely rich in lactic acid. Such feed has a unique smell and easily attracts animals, which could increase the food intake by the animals (Li and Zeng, 2018).

The sauce residue contains about 10 20\% of crude fat and the yeast fermentation process can produce lipase and other digestive enzymes, which can convert the crude fat, non-protein nitrogen, carbohydrates, etc. into microbial protein. In addition, yeast is rich in many essential amino acids, B vitamins and other nutrients and can effectively degrade the anti-nutritional factors (Zhong et al., 2018). It produces coenzyme A, coenzyme I, coenzyme Q, cytochrome C and other biologically active substances (Shi et al., 2017; Zhuang et al., 2020). In this study, we explored symbiotic fermentation by the action of Lactobacillus bulgaricus and Saccharomyces cerevisiae and the effective number of probiotics potentially reached $7.2 \times 10^{8} \mathrm{CFU} \cdot \mathrm{mL}^{-1}$, a result significantly higher than that obtained by (Li et al., 2020), who studied the symbiotic fermentation of palm pulp residue in the presence of beer yeast and Lactobacillus bulgaricus and obtained a total number of colonies equal to $0.9 \times 10^{8} \mathrm{CFU} / \mathrm{g}$. Therefore, the current study showed that the sauce residue is a basic nutrient more suited to symbiotic fermentation in the presence of Lactobacillus bulgaricus and Saccharomyces cerevisiae. Li et al. also showed that when the fermentation temperature was $30^{\circ} \mathrm{C}$, the cell growth and metabolism of the palm kernel residue fermented by the action of beer yeast and Lactobacillus bulgaricus were strongest and the total number of colonies was highest. But the optimal temperature for constant-temperature fermentation in the current study was $32^{\circ} \mathrm{C}$. This may because the different matrix composition led to a slight deviation in the optimum temperature for symbiotic fermentation. In addition to the nutrient composition of sauce residue, which limits the increase in the number of probiotics, yeast growth could produce fatty acids and other substances by metabolize that could inhibit the growth of Lactobacillus bulgaricus. Meanwhile, Lactobacillus bulgaricus could produce lactic acid and other substances during its growth process, which inhibits the growth of yeast and decreases the effective viable count of probiotics during mixed fermentation in the presence of Lactobacillus bulgaricus and Saccharomyces cerevisiae.

A carbon source is essential for the growth and metabolism of Lactobacillus bulgaricus and yeast and it provides the material structure and energy for cell growth and metabolism (Jiang et al., 2018). But sauce residue only contains about $20 \%$ of crude fiber and $10 \%$ of starch. Lactobacillus bulgaricus can convert these macromolecular substances such as starch and protein into small molecular substances like glucose, amino acids and peptides, which are then used by yeast. But the quantity of the small molecular substances often does not meet the needs of microbial growth. Therefore, it is very important to add fermentable sugar that can be directly used by yeast. In this study, it was found that the addition of $40 \mathrm{~g} / \mathrm{L}$ glucose/lactose (1:1) as the carbon source during sauce residue fermentation had the best effect. In fact, many studies have found that monosaccharide glucose was the best carbon source for 
yeast growth (Mao et al., 2018). However, Lactobacillus bulgaricus can make better use of lactose decomposition into 1 molecule of glucose and 1 molecule of galactose as the carbon source and can also provide part of the glucose for yeast. This experiment provides theoretical guidance for finding cheap carbon-source raw materials for practical industrial production and raw materials with lactose and glucose as the main substances will be valued.

\section{Conclusion}

In this study, sauce residue was used as raw material to establish a relatively complex inoculation process and temperature-controlled mode using symbiotic fermentation technology; the symbiotic fermentation conditions of the two probiotics used were optimized. The experimental results showed that the probiotic viable count and the protein content of probiotics could be effectively increased by the initial inoculation of Saccharomyces cerevisiae and then of Lactobacillus bulgaricus after $12 \mathrm{~h}$, with $30^{\circ} \mathrm{C}$ as the initial temperature and an increase of $2^{\circ} \mathrm{C}$ every $12 \mathrm{~h}$ but not exceeding $37^{\circ} \mathrm{C}$. By optimizing the fermentation parameters, the best fermentation conditions were determined as glucose/lactose (1:1) of concentration 40 $\mathrm{g} / \mathrm{L}$, material-liquid ratio of 1:0.8, inoculation volume of $15 \%$ and fermentation time of $48 \mathrm{~h}$. Under these conditions, the effective viable count of probiotics could reach $7.2 \times 10^{8} \mathrm{CFU} \cdot \mathrm{mL}^{-1}$ and the dry-based crude protein content could reach $38.86 \%$. This provides the theoretical and data support for the high-value use of sauce residue to produce high-protein probiotic feed.

\section{Acknowledgement}

The authors are grateful to the funding support by Chongqing technological innovation and application development key projects (cstc2019jscx-gksbX0113), China Postdoctoral Science Foundation, China (2019M662362 and 2019M650167), Shandong key research and development program-Major scientific and technological projects, China (2019JZZY011005), Innovation Project Special Fund for Post Doctors of Shandong Province, China (201902048).

\section{Author's Contributions}

Xinhe Zhao and Zhuangzhuang: Contributed equally and participated in the whole experiment process and also contributed to the interpretation of the results and manuscript preparation.

Wenwei Lu and Yubin Zhao: Participated in part of the experimental design and manuscript preparation. Yuanda Song: Ameliorated the manuscript.

Yuanda Song and Xiaojie Ren: Contributed to the guidance of experimental design and ameliorated the manuscript.

\section{Ethics}

This article is original and contains unpublished material. The corresponding author confirms that all of the other authors have read and approved the manuscript and no ethical issues involved.

\section{References}

Asada, C., Kondo, Y., Sasaki, C., \& Nakamura, Y. (2010). Bioconversion of soy sauce residue treated with steam explosion into ethanol by Meicelase and Mucor indicus. Journal of Food Technology, 8(4), 187-190.

Barton, M. D. (2000). Antibiotic use in animal feed and its impact on human healt. Nutrition research reviews, 13(2), 279-299.

Bian, M., Zong, X., \& Zuo, Y. (2012). Process optimization of synergistic fermentation using sauce residue for protein feed. Feed Research. 08, 83-85.

Chen, P., Song, H., Wang, Y., Chen, P., Shen, X., \& Yao, S. (2015). Homogeneous acetylation of hemicelluloses from soy sauce residue in imidazolium-based ionic liquid. Journal of Material Cycles and Waste Management, 17(3), 574-582.

Chen, X., Luo, Y., Qi, B., Luo, J., \& Wan, Y. (2017). Improving the hydrolysis efficiency of soy sauce residue using ultrasonic probe-assisted enzymolysis technology. Ultrasonics Sonochemistry, 35, 351-358.

Chen, X., Luo, Y., Qi, B., \& Wan, Y. (2014). Simultaneous extraction of oil and soy isoflavones from soy sauce residue using ultrasonic-assisted two-phase solvent extraction technology. Separation and Purification Technology, 128, 72-79.

Cui, Y., Dong, X., \& Tong, J. (2014). Application of Residue Products of Food and other Manufacture as Feed Resource in China. Chinese Journal of Animal Nutrition, (7), 4.

Duan, Y., Zhou, A., Wen, K., Liu, Z., Liu, W., Wang, A., \& Yue, X. (2019). Upgrading VFAs bioproduction from waste activated sludge via co-fermentation with soy sauce residue. Frontiers of Environmental Science \& Engineering, 13(1), 3.

Gong, S., Liu, C. L., \& Shu, X. G. (2018). Research Progress on Refine Utilization of Soy Sauce Residue. Journal of Anhui Agricultural Sciences, 2018(15), 9.

Gu, L., H, J., \& Peng, W. (2015). Add the Impact of Antibiotics on Intestinal Health of Animals.

Han, X., Lei, P., \& Qiao, F. (2014). Research progress on symbiotic between yeasts and Lactic Acid Bacteria in fermented dairy products. Science and Technology of Food Industry. 35(07), 388-391.

He, H. F., Yan, J., Lin, H. L., \& Liu, Q. H. (2014). Extracting crude fat from soy sauce residue. Food and Fermentation Industries, (10), 38. 
Hou, N., Xie, Q., \& Lei, C. (2018). Effects of microbial solid-state fermentation on nutritional quality of soybean meal. China Feed. 5), 32-34.

Jiang, Y., Pan, T., \& Xi, Z. (2018). Effect of assimilable nitrogen and reducing sugar concentrations of synthetic grape must on the fermentation characteristics of Saccharomyces cerevisiae. Shipin Kexue/Food Science, 39(2), 131-137.

Jing, X., Liu, Y., \& Lin, H. (2016). Maize growth influenced by conjugated sauce residue oil-coated fertilizer and coating structure analysis. Jiangsu Journal of Agricultural Sciences. 32(03), 563-569.

Kumar, K., Gupta, S. C., Baidoo, S. K., Chander, Y., \& Rosen, C. J. (2005). Antibiotic uptake by plants from soil fertilized with animal manure. Journal of environmental quality, 34(6), 2082-2085.

Li, B., Jia, H., \& Peer, M. A. (2020). Response surface optimization of preparing palm residues feed by fermention with Saccharomyces cerevisiae and Lactobacillus. China Oils and Fats. 45(03), 126-134.

Li, H., \& Zeng, Y. (2018). The application of Lacticacid bacteria in solid fermentation feed. China Feed. 17, 86-89.

Mao, X., Xia, Y., \& Zhang, Y. (2018). Isolation, identification and fermentation characteristics of yeast from Sichuan bran vinegar starter. Journal of Food Science and Technology. 36(06), 21-27.

Murasawa, N., Koseki, H., \& Iwata, Y. (2013). Causes of accidents by soy sauce squeezing residue and fish meal. Journal of Material Cycles and Waste Management, 15(1), 42-48.

Peng, L., Liang, M., Huan, F., \& Wenjie, W. (2013). Study on degradation of soy sauce residues by Aspergillus niger. Feed Industry, 2013(16), 10.

Shi, Y., Du, W., \& Yao, J. (2017). Progress in extracting coenzyme Q1 0 produced by yeast fermentation. Biotic Resources. 39(03), 168-174.

Shu, D., Wang, D., \& Song, X. (2015). Extraction methods and process optimization of soy sauce residue protein. China Brewing. 34(06), 67-71.
Sun, X., Meng, X., \& Su, J. (2016). Research Progress on Application and Safety of Soybean Isoflavone in Animal Husbandry. China Animal Husbandry \& Veterinary Medicine. 43(08), 2208-2213.

Wang, Y., Xie, H., \& Zhang, N. (2019). Application and development expectation of microbial fermentation feed. Journal of Henan Institute of Science and Technology. 47(06), 49-53.

Yan, B., \& He, Y. F. (2012). Symbiotic fermentation characteristics of lactic acid bacteria and yeast isolated from koumiss in Inner Mongolia. Food Science, 7, 1311137.

Yu, X., Lv, J., \& Yu, F. (2018). Research on Antioxidant Enzyme Activities of Lactic Acid Bacteria in Different Media. China Condiment. 43(01), 39-42.

Zhai, Y., Zhang, H., \& Zhang, L. (2018). Preparation of Biological Feed with Grape Pomace Fermented by Yeast and Lactobacillus. Animal Husbandry and Feed Science. 39(07), 70-75.

Zhan, T., Ke, F., \& Chen, Q. (2015). Effects of fermentation time and material-water ratio on soybean meal fermentation. Journal of Fujian Agriculture and Forestry University. 44(02), 193-197.

Zhang, J., Yuan, J., Zhang, W. X., Zhu, W. Y., Tu, F., Jiang, Y., \& Sun, C. Z. (2017). An aerobic detoxification photofermentation by Rhodospirillum rubrum for converting soy sauce residue into feed with moderate pretreatment. World Journal of Microbiology and Biotechnology, 33(10), 184.

Zhao, X., \& Dai, X. (2020). The mechanism of probiotics'function and its application in animal husbandry. China Feed. 06, 21-23.

Zhong, W., Qi, H., \& Zhao, Y. (2018). Application of Yeast Preparation to Regulate Ruminant Production and Rumen Ecology. Chinese Journal of Animal Science. 54(08), 26-30.

Zhuang, R., Wang, R., \& Qiu, X. (2020). Bioactive substances produced by Rhodotorula mucilaginosa: a comprehensive review. Food Science. 41(01), 318-329. 